\title{
Utilização de medicamentos entre crianças de zero a seis anos: um estudo de base populacional no sul do Brasil
}

\author{
Drug use among children between zero and six years old: \\ a population baseline study in the south of Brazil
}

Cassia Garcia Moraes ${ }^{1}$

Sotero Serrate Mengue ${ }^{1}$

Noemia Urruth Leão Tavares ${ }^{1}$

Tatiane da Silva Dal Pizzol ${ }^{1}$
${ }^{1}$ Programa de Pós-

Graduação em

Epidemiologia,

Universidade Federal do Rio

Grande do Sul. R. Ramiro

Barcelos 2400/2, Santa

Cecília. 90.035-003 Porto

Alegre RS Brasil.

cassia.moraes@ufrgs.br
Abstract The scope of this article was to estimate the prevalence of medication use in children between zero and six years old, analyzing the associated socio-demographic characteristics, and evaluating the adequacy of the medication with respect to pediatric recommendations and restrictions per age group. A cross-sectional study was conducted by means of cluster sampling on a sample comprised of children aged six or under, residents of a city in the south of Brazil. A standardized questionnaire about medication use 15 days prior to the interview was applied. A descriptive analysis was carried out, and the association between medication use and socio-demographic factors was evaluated, as well as the analysis of the pediatric adequacy of the most prevalent medication. Of the 687 children evaluated, 52\% used at least one drug in the period. Associations between medication use and socio-demographic characteristics were not found, with the exception of per capita monthly income. The most prevalent medication was paracetamol (17.1\%), followed by amoxicillin (9.5\%) and dipyrone (8.4\%). Among the ten drugs most used in children, six had pediatric restrictions for the age group. The results indicate significant use of medication, including medication with age restrictions, particularly for children under two years of age.

Key words Drug utilization, Preschool child, Prevalence
Resumo O objetivo do artigo é estimar a prevalência de utilização de medicamentos em crianças de zero a seis anos, analisando as características sociodemográficas associadas, e avaliar a adequação dos medicamentos quanto às indicações em pediatria e às restrições por faixa etária. Estudo transversal de amostra constituída por crianças de seis anos ou menos residentes em uma cidade no sul do Brasil, através de amostragem por conglomerados. Aplicou-se questionário padronizado sobre o uso de medicamentos nos 15 dias anteriores à entrevista. Foi realizada análise descritiva e avaliada a associação entre o uso de medicamentos e fatores sociodemográficos, além da análise da adequação dos medicamentos mais prevalentes. Das 687 crianças avaliadas, 52\% utilizaram pelo menos um medicamento no período estudado. Não foram encontradas associações entre o uso de medicamentos e características sociodemográficas, à exceção da renda per capita mensal. O medicamento mais utilizado foi o paracetamol (17,1\%), seguido da amoxicilina (9,5\%) e da dipirona $(8,4 \%)$. Entre os dez medicamentos mais utilizados, seis apresentaram restrições de faixa etária em pediatria. Os resultados indicam uso expressivo de medicamentos, incluindo os com restrições de faixa etária, particularmente em crianças menores de dois anos.

Palavras-chave Uso de medicamentos, Crianças, Prevalência 


\section{Introdução}

A incerteza quanto à eficácia e segurança de medicamentos utilizados em crianças deve-se, em grande parte, à escassez de ensaios clínicos envolvendo estas por motivos éticos, legais e econômicos, limitando o conhecimento sobre os efeitos do medicamento no organismo infantil ${ }^{1-3}$. Como consequência, o uso de medicamentos em crianças é baseado principalmente em extrapolações e adaptações do uso em adultos. Desta forma, muitos medicamentos são prescritos e dispensados às crianças, mesmo quando o uso não está aprovado pela agência sanitária ou quando apresenta riscos pela ausência de informações para uso pediátrico ${ }^{1,4,5}$.

Os estudos sobre o padrão de utilização de medicamentos em crianças ainda são considerados escassos, sobretudo os de base populacional e os realizados nos países em desenvolvimento ${ }^{4,6,7}$. Os estudos existentes apontam que a maioria das crianças utiliza algum tipo de medicamento, principalmente as menores de dois anos ${ }^{8-10}$. Estudo realizado nos Estados Unidos indica uma prevalência de consumo de $56 \%$ nos sete dias anteriores à pesquisa ${ }^{11}$. Outro estudo, conduzido no sul do Brasil, verificou a mesma frequência de uso, mas para os 15 dias anteriores à entrevista ${ }^{6}$.

A investigação sobre o perfil de uso dos medicamentos pode fornecer uma visão geral sobre os problemas terapêuticos, ajudando a identificar as necessidades de intervenções específicas junto à população e aos profissionais da saúde ${ }^{12}$, bem como de pesquisas clínicas e/ou epidemiológicas com os medicamentos de uso prevalente. Neste contexto, estudos farmacoepidemiológicos de base populacional podem ser úteis, visando melhorias na eficácia e eficiência das intervenções em saúde ${ }^{13}$.

Com o intuito de realizar contribuições nessa área, foi realizado um estudo epidemiológico de base populacional em crianças de zero a seis anos residentes na área urbana da cidade de Bagé, situada na região sul do Brasil. O objetivo foi estimar a prevalência de utilização de medicamentos, analisando as características sociodemográficas associadas, e avaliar a adequação dos medicamentos quanto às indicações em pediatria $\mathrm{e}$ restrições por faixa etária.

\section{Métodos}

O estudo foi realizado na área urbana de Bagé, cidade no sul do Brasil. Em 2000 havia 114.908 pessoas residentes no município, das quais 14.205 eram crianças entre zero e seis anos. O índice de desenvolvimento humano municipal (IDHM), em 2000, era de 0,802, acima do índice nacional $(0,766)^{14}$.

A pesquisa seguiu um modelo de estudo transversal. A amostra foi constituída por crianças entre zero e seis anos residentes em domicílios da área urbana de Bagé no período de 13 de abril a 25 de maio de 2009. Como critérios de inclusão, foram selecionadas crianças nascidas após 13 de abril de 2003. Os critérios de exclusão foram crianças com problemas graves de saúde e cuidadores com dificuldades de comunicação.

O tamanho amostral calculado para se estimar uma prevalência, presumida em 55\%, obtida a partir de estudos prévios, com um erro absoluto de 0,05 , ou seja, um afastamento tolerável de $5 \%$ entre a prevalência na população e a estimativa a ser obtida pelo estudo, e para um nível de confiança de $95 \%$, foi de 384 crianças. Por meio de amostragem aleatória simples, foram sorteados 30 setores censitários. As quadras de cada setor foram numeradas e a primeira a ser percorrida sorteada, com uma esquina de ponto de partida também escolhida por sorteio. O entrevistador abordava todas as casas procurando por crianças nascidas após 13 de abril de 2003 até atingir o total de 25 crianças por setor. Havendo duas ou mais crianças elegíveis no mesmo domicílio, era sorteada uma das crianças para a qual os dados eram coletados.

Foram convidados a responder o questionário os pais ou o cuidador da criança, correspondendo ao principal responsável pelas decisões em relação às condutas com a saúde da criança. $\mathrm{Na}$ dúvida, perguntava-se "Quem é a pessoa da casa que toma as decisões sobre os cuidados com a saúde da criança, na maioria das vezes"? No caso de empate entre mãe e outra pessoa, escolhia-se preferencialmente a mãe. Caso o responsável pela criança não estivesse presente no dia da entrevista agendava-se nova visita em horário conveniente. Caso não fosse possível realizar a entrevista ou agendá-la após três tentativas de contato em dias e horários diferentes, o sujeito era considerado como perda.

Utilizamos um questionário contendo perguntas abertas e fechadas, testado em estudo piloto, fase em que foram realizadas as modificações necessárias. Os entrevistadores foram previamente treinados para os procedimentos relacionados com a localização dos domicílios sorteados, abordagem dos moradores, convite à participação no projeto e aplicação do questionário. 
O uso de medicamentos, por prescrição ou por automedicação, foi verificado por meio da questão: "A criança recebeu algum remédio nos últimos 15 dias, inclusive vitaminas, chás ou pomadas?". Em caso afirmativo, eram coletados dados sobre nome comercial e/ou genérico do medicamento, forma farmacêutica e posologia. Os medicamentos citados foram ordenados de acordo com a classificação Anatomical Therapeutic Chemical (ATC).

As principais variáveis independentes investigadas foram sexo da criança, idade, número de irmãos e cuidador, e características do responsável pela criança (raça autorreferida, anos de escolaridade, idade e ocupação relacionada ao exercício remunerado ou não). Foi utilizada a classificação econômica familiar, por meio de estimativa do poder de compra da família (de acordo com o Critério de Classificação Econômica Brasil, da Associação Brasileira de Empresas de Pesquisa), a renda mensal per capita em reais e o plano de saúde familiar (se privado ou público). A principal variável de desfecho investigada foi a utilização de medicamentos, prescritos ou não. Os mais utilizados foram avaliados quanto às indicações aprovadas em pediatria e restrições por faixa etária, de acordo com as informações verificadas a partir da base de dados Drugdex Thomsom Micromedex e da Agência Nacional de Vigilância Sanitária (ANVISA).

Os dados foram digitalizados pelo Teleform Workgroup V10, armazenados e analisados no programa estatístico SPSS versão 18.0 para Windows (SPSS Inc, Chicago, IL). Foi realizada análise descritiva e os dados expressos em frequência relativa e razão de prevalência (RP), com intervalos de confiança de 95\%. O teste do qui-quadrado foi usado para comparação bivariada de proporções, com um valor de $\mathrm{P}<0,05$ sendo considerado significativo.

O presente estudo foi submetido ao Comitê de Ética em Pesquisa da Universidade Federal do Rio Grande do Sul, tendo sido aprovado. O termo de consentimento livre e esclarecido era lido e assinado no momento da pesquisa pelas partes, em duas vias.

\section{Resultados}

De um total de 693 crianças identificadas, ocorreram seis perdas por recusa ou residência fechada em três tentativas de contato. Em cinco setores censitários, não foram encontradas o total de 25 crianças que preenchessem os critérios de inclusão. Foram analisadas 687 crianças, correspondendo a $4,8 \%$ da população entre zero e seis anos de Bagé, em $2000^{15}$.

Das 687 crianças incluídas no estudo, o uso de pelo menos um medicamento nos 15 dias anteriores à entrevista foi relatado para 357 crianças (52\%, IC 95\%: 46,9 - 57,0), por indicação médica ou por automedicação. O total de medicamentos utilizados no período foi igual a 597, sendo a média por criança de 1,7 (DP $\pm 1,0)$. Observou-se que $54,7 \%$ das crianças menores de dois anos participantes do estudo utilizaram medicamentos nos 15 dias anteriores à entrevista, enquanto $50,6 \%$ daquelas com dois anos ou mais utilizaram algum medicamento no mesmo período.

A Tabela 1 apresenta as prevalências e RP de uso de medicamentos, de acordo com as características sociodemográficas das crianças e de seus cuidadores. Renda per capita mensal maior que um salário mínimo apresentou associação positiva com uso de medicamentos $(\mathrm{p}<0,05)$. Para as demais características das crianças e cuidadores investigadas, não foram encontradas diferenças entre as usuárias e não usuárias de medicamento.

Os grupos terapêuticos mais utilizados pelas crianças, conforme o primeiro nível da classificação ATC, foram os medicamentos com ação no sistema respiratório, utilizado por cerca de um terço das crianças, seguido pelos que atuam no sistema nervoso central (28,0\%) (Tabela 2$)$. A Tabela 2 ainda mostra os medicamentos mais usados por grupo terapêutico. Os chás e os produtos homeopáticos foram utilizados por 24,1\% das crianças, entre os quais o de laranjeira, camomila e erva-doce foram os mais citados. O medicamento mais utilizado foi o paracetamol $(17,1 \%)$, seguido da amoxicilina $(9,5 \%)$ e da dipirona $(8,4 \%)$.

Entre os dez medicamentos mais utilizados pelas crianças no estudo, seis apresentam restrições de faixa etária em pediatria. Os medicamentos e as faixas etárias com contraindicação são apresentados na Tabela 3.

\section{Discussão}

Aproximadamente metade das crianças de zero a seis anos incluídas no estudo utilizou ao menos um medicamento nos 15 dias anteriores à entrevista, por indicação médica ou não. A prevalência estimada é semelhante a outros estudos brasileiros que usaram o mesmo período recordatório, nos quais variou de 48 a $56 \%{ }^{4,6}$. Estudos realizados nos Estados Unidos ${ }^{11}$ e Alemanha ${ }^{16}$ 
Tabela 1. Prevalência e razão de prevalências (RP) de uso de medicamentos, de acordo com características sociodemográficas das crianças e de seus cuidadores (687).

\begin{tabular}{|c|c|c|c|c|c|}
\hline & n total & $\%$ uso & IC 95\% & $\mathbf{R} \mathbf{P}$ & IC 95\% \\
\hline Entrevistados & 687 & 52 & $(46,9-57,0)$ & & \\
\hline \multicolumn{6}{|c|}{ Características das Crianças } \\
\hline \multicolumn{6}{|c|}{ Sexo } \\
\hline Feminino & 347 & 50,1 & $(44,1-56,2)$ & 0,93 & $(0,82-1,05)$ \\
\hline Masculino & 340 & 53,8 & $(47,9-59,6)$ & 1,00 & \\
\hline \multicolumn{6}{|l|}{ Idade } \\
\hline$<2$ anos & 212 & 54,7 & $(46,3-62,8)$ & 1,00 & \\
\hline$\geq 2$ anos & 474 & 50,6 & $(44,8-56,6)$ & 0,92 & $(0,77-1,11)$ \\
\hline \multicolumn{6}{|l|}{ Número de irmãos } \\
\hline 0 & 255 & 53,7 & $(46,6-60,7)$ & 1,00 & \\
\hline$\geq 1$ & 410 & 52,4 & $(46,1-58,7)$ & 0,98 & $(0,82-1,17)$ \\
\hline \multicolumn{6}{|l|}{ Cuidador } \\
\hline Pais & 602 & 50,7 & $(45,8-55,5)$ & 0,83 & $(0,69-1,02)$ \\
\hline Outros & 84 & 60,7 & $(47,2-72,8)$ & 1,00 & \\
\hline \multicolumn{6}{|c|}{ Características do Cuidador } \\
\hline \multicolumn{6}{|c|}{ Raça autorreferida } \\
\hline Branca & 534 & 51,5 & $(46,7-56,2)$ & 0,97 & $(0,79-1,19)$ \\
\hline Não Branca & 152 & 53,3 & $(42,0-64,3)$ & 1,00 & \\
\hline \multicolumn{6}{|l|}{ Escolaridade } \\
\hline 0 a 8 anos & 284 & 50,4 & $(41,8-58,9)$ & 1,00 & \\
\hline 9 anos ou mais & 400 & 53,3 & $(48,7-57,7)$ & 1,06 & $(0,89-1,26)$ \\
\hline \multicolumn{6}{|l|}{ Trabalha fora } \\
\hline Sim & 277 & 53,1 & $(47,2-58,8)$ & 1,02 & $(0,89-1,17)$ \\
\hline Não & 394 & 52,0 & $(45,6-58,4)$ & 1,00 & \\
\hline \multicolumn{6}{|l|}{ Idade, em anos } \\
\hline 13 a 29 & 293 & 50,5 & $(44,0-57,0)$ & 1,00 & \\
\hline 30 a 39 & 252 & 54,0 & $(48,5-59,3)$ & 1,07 & $(0,92-1,23)$ \\
\hline 40 a 79 & 140 & 51,4 & $(40,5-62,2)$ & 1,02 & $(0,81-1,27)$ \\
\hline \multicolumn{6}{|l|}{ Classe Econômica } \\
\hline $\mathrm{A}$ e $\mathrm{B}$ & 229 & 55,9 & $(49,4-62,2)$ & 1,14 & $(0,97-1,34)$ \\
\hline C, D e E & 427 & 48,9 & $(42,5-55,5)$ & 1,00 & \\
\hline \multicolumn{6}{|c|}{ Renda mensal per capita* } \\
\hline Até um $\mathrm{SM}^{* *}$ & 478 & 49,2 & $(43,3-55,1)$ & 1,00 & \\
\hline Mais de um SM & 200 & 59,0 & $(52,1-65,5)$ & 1,20 & $(1,03-1,40)$ \\
\hline \multicolumn{6}{|l|}{ Plano de Saúde } \\
\hline Sim & 279 & 54,5 & $(48,6-60,2)$ & 1,07 & $(0,92-1,24)$ \\
\hline Não & 396 & 51,0 & $(44,5-57,5)$ & 1,00 & \\
\hline
\end{tabular}

"teste do qui-quadrado, p<0,05; ** SM = salário mínimo, correspondendo a 465 reais, valor vigente em 2009.

também demonstraram prevalências semelhantes, utilizando período recordatório de sete dias. A heterogeneidade dos métodos utilizados em outros estudos dificulta a comparação dos dados, visto que a faixa etária investigada e o período recordatório têm grande variação nos diferentes trabalhos realizados, bem como a origem do uso dos medicamentos. Enquanto alguns estudos investigaram o uso de medicamentos por indicação médica ${ }^{1}$ outros avaliaram o uso por automedicação $0^{5,7}$.
No presente estudo, as variáveis relacionadas às características sociodemográficas das crianças e seus responsáveis não apresentaram associação com o consumo de medicamento, à exceção da renda per capita mensal, tendo em vista que uma maior renda familiar aumenta a possibilidade de aquisição tanto de produtos como de serviços ${ }^{16}$.

Os medicamentos com ação no trato respiratório formam o grupo terapêutico mais utilizado pelas crianças no estudo, em consonância 
Tabela 2. Grupos terapêuticos de acordo com a classificação ATC nos níveis 1 e 2 e medicamentos mais utilizados pelas crianças nos 15 dias anteriores à entrevista.

\begin{tabular}{|c|c|c|c|c|c|c|}
\hline & ATC Níveis 1 e 2 & $\begin{array}{c}\% \\
\text { Medicamentos } \\
(597)\end{array}$ & $\begin{array}{c}\% \\
\text { Uso } \\
\left(357^{*}\right)\end{array}$ & $\begin{array}{l}\text { Medicamentos } \\
\text { mais usados } \\
\text { por grupo }\end{array}$ & $\begin{array}{c}\% \\
\text { Medicamentos } \\
(597)\end{array}$ & $\begin{array}{c}\% \\
\text { Uso } \\
\left(357^{*}\right)\end{array}$ \\
\hline A00 & Trato alimentar e metabolismo & 12,9 & 21,6 & & & \\
\hline \multirow[t]{3}{*}{ A 11} & Vitaminas & 7,5 & 12,6 & Multivitaminas & 3,2 & 5,3 \\
\hline & & & & Vitamina $A+D$ & 2,0 & 3,4 \\
\hline & & & & Vitamina $C$ & 1,5 & 2,5 \\
\hline D00 & Dermatológicos & 8,5 & 14,3 & & & \\
\hline D01 & Antifúngicos de uso dermatológico & 2,0 & 3,4 & Nistatina & 1,7 & 2,8 \\
\hline D07 & $\begin{array}{l}\text { Corticoesteróides de uso } \\
\text { dermatológico }\end{array}$ & 2,7 & 4,5 & $\begin{array}{l}\text { Hialuronidase }+ \\
\text { betametasona }\end{array}$ & 0,8 & 1,4 \\
\hline D09 & Medicamentos curativos & 1,8 & 3,1 & $\begin{array}{l}\text { Óxido de } \\
\text { Zinco+vit. A e D }\end{array}$ & 1,8 & 3,1 \\
\hline $\mathrm{J} 00$ & Antiinfecciosos de uso sistêmico & 11,7 & 19,6 & & & \\
\hline \multirow[t]{3}{*}{ J01 } & Antibacterianos & 11,7 & 19,6 & Amoxicilina & 5,7 & 9,5 \\
\hline & & & & Cefalexina & 2,0 & 3,4 \\
\hline & & & & $\begin{array}{l}\text { Sulfametoxazol + } \\
\text { trimetoprima }\end{array}$ & 1,3 & 2,3 \\
\hline M00 & Sistema músculo-esquelético & 7,4 & 12,3 & & & \\
\hline \multirow[t]{3}{*}{ M01 } & Antiinflamatórios e antireumáticos & 7,0 & 11,8 & Ibuprofeno & 4,2 & 7,0 \\
\hline & & & & Diclofenaco & 2,0 & 3,4 \\
\hline & & & & Nimesulida & 0,5 & 0,8 \\
\hline N00 & Sistema nervoso & 16,8 & 28,0 & & & \\
\hline \multirow[t]{3}{*}{ N02 } & Analgésicos & 16,6 & 27,7 & Paracetamol & 10,2 & 17,1 \\
\hline & & & & Dipirona sódica & 5,0 & 8,4 \\
\hline & & & & $\begin{array}{l}\text { Ácido } \\
\text { acetilsalicílico }\end{array}$ & 1,2 & 2,0 \\
\hline R00 & Sistema respiratório & 19,8 & 33,0 & & & \\
\hline R01 & Preparações Nasais & 4,7 & 7,8 & $\begin{array}{l}\text { Bronfeniramina }+ \\
\text { Fenilefrina }\end{array}$ & 4,0 & 6,7 \\
\hline R05 & Produtos para tosse e expectorantes & 4,7 & 7,8 & Bromexina & 2,2 & 3,6 \\
\hline R06 & Antihistamínicos (uso sistêmico) & 7,0 & 11,8 & Dexclorfeniramina & 3,7 & 6,0 \\
\hline V00 & Vários & 14,6 & 24,4 & & & \\
\hline V03 & Outros produtos terapêuticos & 14,4 & 24,1 & $\begin{array}{l}\text { Chás e } \\
\text { homeopatias }\end{array}$ & 14,4 & 24,1 \\
\hline $\begin{array}{l}\text { Outros } \\
\text { H00, I }\end{array}$ & $\begin{array}{l}\text { s grupos terapêuticos } \quad(B 00, C 00 \text {, } \\
\mathrm{L} 00, \mathrm{P} 00, \mathrm{~S} 00)^{* *}\end{array}$ & 8,4 & 14,0 & & & \\
\hline
\end{tabular}

*nenhuma criança utilizou o mesmo fármaco mais de uma vez. ${ }^{\star *}$ Outros grupos terapêuticos (B00: sangue e órgãos hematopoiéticos, C00: sistema cardiovascular, H00: preparados hormonais sistêmicos, L00: antineoplásicos e imunomoduladores, P00: antiparasitários, S00: órgãos sensoriais)

com pesquisas prévias sobre o uso destes, seja por prescrição médica ${ }^{1}$ ou por automedicaçã $0^{5}$.

Entre os medicamentos com ação no trato respiratório, os mais utilizados foram os antihistamínicos, fármacos para tosse e expectorantes e as preparações nasais. Diversas revisões sistemáticas têm revelado que não existem evidências suficientes de que estes medicamentos apresentam benefício superior ao placebo no tratamento de sintomas gerados por infecções respi- ratórias das vias áreas superiores, como congestão nasal e rinorreia associados a resfriado comum $^{17,18}$ tosse aguda ${ }^{19,20}$, tosse crônica inespecífica $^{21}$ e sinusite aguda ${ }^{22}$.

Embora alguns dos medicamentos para o trato respiratório como dexclorfeniramina e a associação bronfeniramina e fenilefrina sejam contraindicados para crianças menores de dois anos, verificamos que cerca de um terço das que usaram esses medicamentos estavam nesta faixa 
Tabela 3. Informações sobre o uso e a faixa etária de contraindicação dos medicamentos mais utilizados pelas crianças de zero a seis anos durante o período de estudo.

\begin{tabular}{lccc}
\hline \multicolumn{1}{c}{$\begin{array}{c}\text { Medicamentos } \\
\text { mais frequentes }\end{array}$} & $\begin{array}{c}\text { Faixa etária } \\
\text { contraindicada }\end{array}$ & $\begin{array}{c}\text { Crianças que } \\
\text { usaram (n) }\end{array}$ & $\begin{array}{c}\text { Crianças com idade } \\
\text { contraindicada (\%) }\end{array}$ \\
\hline Paracetamol & - & 61 & - \\
Amoxicilina & -- & 34 & - \\
Dipirona sódica & $<3$ meses & 30 & 0,0 \\
Ibuprofeno & $<6$ meses & 25 & 12,0 \\
Bronfeniramina + Fenilefrina & $<2$ anos & 24 & 29,2 \\
Dexclorfeniramina & $<2$ anos & 22 & 27,3 \\
Multivitaminas & -- & 19 & - \\
Bromexina & $<2$ anos & 13 & 15,4 \\
Diclofenaco* & $<1$ ano & 12 & 33,3 \\
Cefalexina & -- & 12 & - \\
\hline
\end{tabular}

‘Na faixa etária entre um e quatorze anos, o diclofenaco é indicado apenas para o tratamento de artrite juvenil crônica.

etária ${ }^{23,24}$. Além dos efeitos adversos intrínsecos de cada substância ativa, existem outros fatores que podem torná-los potencialmente perigosos para esse grupo etário, incluindo a interpretação incorreta da dose ou do intervalo entre doses, o uso de medidas inadequadas de dosificação, ou ainda a administração simultânea de vários medicamentos, com o intuito de obter maior alívio dos sintomas.

Entre os analgésicos e anti-inflamatórios, os mais utilizados foram paracetamol, dipirona e ibuprofeno, fármacos reconhecidamente eficazes no manejo da febre, um dos sintomas mais comuns em pediatria ${ }^{25}$. O paracetamol e o ibuprofeno estão na lista de medicamentos para infância da Organização Mundial de Saúde ${ }^{26}$. A segurança da dipirona, analgésico/antitérmico de baixo custo e integrante da lista de medicamentos do Programa Farmácia Popular, tem sido questionada em várias partes do mundo. Resultados do Latin study, estudo de caso-controle multicêntrico realizado em sete locais do Brasil, dois na Argentina e outro no México, apontam para baixa incidência de anemia aplastica (1,6 casos por 1 milhão de habitantes/ano) e ausência de associação com dipirona ${ }^{27}$, e incidência extremamente baixa de agranulocitose $(0,38$ casos por 1 milhão de habitantes/ano) e associação positiva, embora não estatisticamente significativa, com dipirona $(\mathrm{OR}=2,4 ; \mathrm{IC} 95 \% 0,8-6,7 ; \mathrm{p}=0,12)^{28}$. Por outro lado, estudo sobre a qualidade dos produtos contendo dipirona comercializados no Brasil, revela desvios de qualidade no teor do fármaco e nas informações presentes na embalagem e em bulas de medicamentos, principal- mente dos similares ${ }^{29}$, chamando a atenção para a necessidade de maior vigilância, no Brasil.

Destacamos ainda o uso expressivo do diclofenaco em crianças com menos de um ano de idade, faixa etária para o qual o medicamento é contraindicado. A eficácia e a segurança deste fármaco para uso em pediatria não estão estabelecidas $^{24}$.

Outro grupo terapêutico que chama a atenção pelo elevado percentual de utilização corresponde aos chás e produtos homeopáticos, agrupados em "outros produtos terapêuticos". Mesmo que as evidências de segurança ou efetividade de terapias complementares sejam escassas, se comparadas à terapêutica convencional, tais produtos são geralmente considerados seguros e/ou naturais pelos pais, que os administram aos seus filhos com ou sem ciência do médico ${ }^{30,31}$. Ao avaliar essa prática, é importante compreender os aspectos psicológicos e socioculturais que explicam os motivos pelos quais os pais sentem-se estimulados a adotar esses recursos para amenizar o desconforto dos seus filhos.

Os antibióticos representam o terceiro subgrupo mais utilizado pela amostra investigada. Em diversos estudos ${ }^{2,4}$ os antibióticos aparecem na lista dos medicamentos mais utilizados por crianças, principalmente naqueles com medicamentos prescritos ${ }^{10,11}$. Este resultado já era esperado, tendo em vista que as doenças infecciosas também estão entre as morbidades mais comuns em crianças, principalmente as infecções de vias respiratórias, que são responsáveis por grande parte das prescrições ambulatoriais de antimicrobianos ${ }^{9}$. Dentre os antibióticos, a amo- 
xicilina foi o mais utilizado pelas crianças, resultado similar ao verificado por outros investigadores ${ }^{4,10,11}$. A amoxicilina é citada em diretrizes internacionais como primeira escolha de tratamento para as infecções mais comuns da infância, como a otite média aguda, faringo-amigdalite e sinusite ${ }^{3}$.

Outro grupo terapêutico muito utilizado foi o das vitaminas. Há poucas evidências na literatura sobre os benefícios da suplementação vitamínica, principalmente quando não é detectada uma carência destes componentes ${ }^{32}$. Estabelecer precisamente as necessidades diárias de vitaminas é complicado, pois devem ser consideradas as variações individuais, além do fato de que a ingestão de nutrientes recomendados é possível com uma dieta equilibrada ${ }^{33}$.

Todos os medicamentos utilizados para o tratamento de crianças devem ser submetidos ao processo de licenciamento para assegurar a qualidade, a segurança e a eficácia nessa faixa etária. Os quatro medicamentos mais usados na amostra analisada (paracetamol, amoxicilina, dipirona e ibuprofeno) possuem uma boa documentação para o uso pediátrico, embora a indicação não seja recomendada em algumas faixas etárias $^{23,24}$. Por outro lado, os medicamentos para o trato respiratório apresentam poucas evidências de eficácia, conforme discutido anteriormente. Um dos aspectos mais importantes a ser considerado na avaliação das evidências disponíveis em pediatria é como lidar com as questões éticas de proteção da criança na realização de ensaios clínicos controlados. Por definição, estes sempre envolvem algum grau de risco que, na pediatria, deve ser assumido pelos pais, frente a potenciais bene- fícios que não serão imediatamente usufruídos pelos seus próprios filhos. Outro aspecto importante está relacionado com a motivação econômica da indústria farmacêutica para o desenvolvimento de medicamentos para uso pediátrico. $\mathrm{O}$ reduzido mercado dos medicamentos em Pediatria, comparativamente a outros grupos etários, como os adultos e os idosos, além das dificuldades inerentes à realização de ensaios clínicos em crianças, torna o desenvolvimento destes pouco atrativo à indústria farmacêutica ${ }^{34,35}$.

Quanto às limitações deste trabalho, deve-se considerar o viés de memória, já que o uso referido de medicamentos pelos responsáveis das crianças dependia de um período recordatório de 15 dias. A prevalência do uso de alguns medicamentos pode estar subestimada devido à época da realização do estudo, não abrangendo a estação do inverno, onde os problemas respiratórios tornam-se mais frequentes, aumentando a necessidade do consumo de determinados medicamentos ${ }^{23}$.

A prevalência encontrada no presente estudo corrobora os resultados da literatura, que indicam consumo elevado de medicamentos na população infantil, apontando para o uso expressivo daqueles com restrições de indicação e de faixa etária, particularmente para crianças menores de dois anos. A escassez de informações quanto à segurança e eficácia dos medicamentos identificados neste estudo reforça a importância da adoção de medidas de incentivo à realização de pesquisas clínicas e ao desenvolvimento de produtos de uso pediátrico, atendendo aos critérios éticos e de qualidade postulados pelas autoridades sanitárias, profissionais e pesquisadores da área.

\section{Colaboradores}

CG Moraes e TS Dal Pizzol participaram do planejamento e análises dos dados, redação, revisão do manuscrito e aprovação da versão final. NUL Tavares e SS Mengue participaram do planejamento do estudo, revisão do manuscrito e aprovação da versão final. 


\section{Referências}

\section{Agradecimentos}

À equipe de coleta de campo, professora Lúcia Azambuja Saraiva Vieira pela supervisão de campo, aos entrevistadores Bruna Saraiva Severo, Jorge Maubrigades, Lorena da Silva Lima, Luana Lucas Alves, Raquel Leivas Spenst e Raquel Lemos Nobre.
1. Straand J, Rokstad K, Heggedal U. Drug prescribing for children in general practice. A report from the Møre \& Romsdal Prescription Study. Acta Paediatr 1998; 87(2):218-224.

2. Clavenna A, Bonati M. Drug prescriptions to outpatient children: a review of the literature. Eur J Clin Pharmacol 2009; 65(8):749-755.

3. Clavenna A, Berti A, Gualandi L, Rossi E, De Rosa $\mathrm{M}$, Bonati M. Drug utilisation profile in the Italian paediatric population. Eur J Pediatr 2009; 168(2):173180.

4. Santos DB, Barreto ML, Coelho HL. Drug use and associated factors in children living in poor areas. Rev Saude Publica 2009;3(5):768-778.

5. Du Y, Knopf H. Self-medication among children and adolescents in Germany: results of the National Health Survey for Children and Adolescents (KiGGS). Br J Clin Pharmacol 2009; 68(4):599-608.

6. Béria JU, Victora CG, Barros FC, Teixeira AB, Lombardi C. Epidemiology of drug consumption in children of a urban center of the southern region of Brazil. Rev Saude Publica 1993; 27(2):95-104.

7. Pereira FS, Bucaretchi F, Stephan C, Cordeiro R. Self-medication in children and adolescents. $J \mathrm{Pe}$ diatr 2007; 83(5):453-458.

8. Headley J, Northstone K. Medication administered to children from 0 to 7.5 years in the Avon Longitudinal Study of Parents and Children (ALSPAC). Eur J Clin Pharmacol 2007; 63(2):189-195.

9. Oliveira EA, Bertoldi AD, Domingues MR, Santos IS, Barros AJ. Medicine use from birth to age two years: the 2004 Pelotas (Brazil) Birth Cohort study. Rev Saude Publica 2010; 44(4):591-600.

10. Sturkenboom MC, Verhamme KM, Nicolosi A, Murray ML, Neubert A, Caudri D, Picelli G, Sen EF, Giaquinto C, Cantarutti L, Baiardi P, Felisi MG, Ceci A, Wong IC; TEDDY European Network of Excellence. Drug use in children: cohort study in three European countries. BMJ 2008; 337:a2245.

11. Vernacchio L, Kelly JP, Kaufman DW, Mitchell AA. Medication use among children $<12$ years of age in the United States: results from the Slone Survey. Pediatrics 2009; 124(2):446-454.

12. Niclasen BV. Changes in drug prescription over a decade in an Arctic child population. Acta Paediatr 2006; 95(11):1456-1460.

13. Luo X, Doherty J, Cappelleri JC, Frush K. Role of pharmacoepidemiology in evaluating prescription drug safety in pediatrics. Curr Med Res Opin 2007; 23(11):2607-2615.

14. Organização das Nações Unidas (ONU). Programa das Nações Unidas para o Desenvolvimento. Atlas do Desenvolvimento Humano no Brasil. 2003. Indice de Desenvolvimento Humano - Municipal, 1991 e 2000. [site na Internet] 2003. [acessado 2010 out 13]. Diponível em: http://www.pnud.org.br/atlas/ ranking/IDH-M\%2091\%2000\%20Ranking\%20 decrescente $\% 20$ (pelos\%20dados\%20de\%202000).htm 
15. Instituto Brasileiro de Geografia e Estatística (IBGE). População e Domicílios - Censo 2000 com Divisão Territorial 2001, Bagé, RS. [site na Internet] 2010. [acessado 2010 out 2]. Diponível em: http:// www.ibge.gov.br/cidadesat/topwindow.htm? 1

16. Knopf H. Medicine use in children and adolescents. Data collection and first results of the German Health Interview and Examination Survey for Children and Adolescents (KiGGS). Bundesgesundheitsblatt Gesundheitsforschung Gesundheitsschutz. 2007; 50(5-6):863-870.

17. Taverner D, Latte GJ. Withdrawn: Nasal decongestants for the common cold. Cochrane Database Syst Rev 2009(2):CD001953.

18. De Sutter AI, Lemiengre M, Campbell H. Withdrawn: Antihistamines for the common cold. Cochrane Database Syst Rev 2009(4):CD001267.

19. Susan M. Smith KS, Fahey T. Over-the-counter (OTC) medications for acute cough in children and adults in ambulatory settings. The Cochrane Library 2011; 8:CD001831.

20. Schroeder K, Fahey T. Should we advise parents to administer over the counter cough medicines for acute cough? Systematic review of randomised controlled trials. Arch Dis Child 2002; 86(3):170-175.

21. Chang AB, Peake J, McElrea MS. Anti-histamines for prolonged non-specific cough in children. $\mathrm{Co}$ chrane Database Syst Rev 2008(2):CD005604.

22. Shaikh N, Wald ER, Pi M. Decongestants, antihistamines and nasal irrigation for acute sinusitis in children. Cochrane Database Syst Rev 2010(12): CD007909.

23. Pitrez PM, Pitrez JL. Acute upper respiratory tract infections: outpatient diagnosis and treatment. $J$ Pediatr 2003; 79(Supl. 1):S77-S86.

24. DRUGDEX ${ }^{\circledR}$ System. Thomson MICROMEDEX. [site na Internet] 2010. [acessado 2010 out 13]. Disponível em: http://www.thomsonhc.com/home/dispatch

25. Bricks LF, Leone C. Use of medicines by children attending nursery schools. Rev Saude Publica 1996; 30(6):527-535.

26. World Health Organization (WHO). Who model list of essential medicines for children 2010. [site na Internet]. 2010. [acessado 2010 out 13]. Diponível em: http://www.who.int/medicines/publications/essential medicines/Updated_second_children_list_en.pdf

27. Maluf E, Hamerschlak N, Cavalcanti AB , Júnior AA, Eluf-Neto J, Falcão RP, Lorand-Metze IG, Goldenberg D, Santana CL, Rodrigues Dde O, Passos LN, Rosenfeld LG, Pitta M, Loggetto S, Ribeiro AA, Velloso ED, Kondo AT, Coelho EO, Pintão MC, de Souza HM, Borbolla JR, Pasquini R. Incidence and risk factors of aplastic anemia in Latin American countries: the LATIN case-control study. Haematologica 2009; 94(9):1220-1226.
28. Hamerschlak N, Maluf E, Biasi Cavalcanti A, Avezum Júnior A, Eluf-Neto J, Passeto Falcão R, Lorand-Metze IG, Goldenberg D, Leite Santana C, de Oliveira Werneck Rodrigues D, Nascimento da Motta Passos L, Oliveira de Miranda Coelho E, Tostes Pintão MC, Moraes de Souza H, Borbolla JR, Pasquini R. Incidence and risk factors for agranulocytosis in Latin American countries-the Latin Study: a multicenter study. Eur J Clin Pharmacol 2008; 64(9):921-929.

29. Knappmann AL, de Melo EB. Quality of over-thecounter medicines: a study with dipyrone brands commercialized in a drugstore in Cascavel city (Paraná, Brazil). Cien Saude Colet 2010; 15(Supl. 3):34673476.

30. Erlewyn -Lajeunesse LM. Homeopathic medicines for children. Arch Dis Child 2011; 97(2):135-138.

31. Gentil LB, Robles AC, Grosseman S. Use of complementary therapies by mothers in their children: study at an university hospital. Cien Saude Colet 2010; 15(Supl. 1):1293-1299.

32. Allen LH. Nutritional influences on linear growth: a general review. Eur J Clin Nutr 1994; 48(Supl. 1):S75-S89.

33. Leaf AA, Nutrition RSCo. Vitamins for babies and young children. Arch Dis Child 2007; 92(2):160164.

34. Costa PQ, Rey LC, Coelho HL. Lack of drug preparations for use in children in Brazil. J Pediatr 2009; 85(3):229-235.

35. Dinah D, Helena F. Melhores medicamentos em Pediatria. Acta Pediátrica Portuguesa 2008; 39(1):1722.

Artigo apresentado em 03/08/2012

Aprovado em 09/09/2012

Versão final apresentada em 19/10/2012 
